\title{
Dispersion in the Wake of a Rectangular Building: Validation of Two Reynolds-Averaged Navier-Stokes Modelling Approaches
}

\author{
Catherine Gorlé · Jeroen van Beeck • Patrick Rambaud
}

Received: 12 October 2009 / Accepted: 15 June 2010 / Published online: 1 July 2010

(C) Springer Science+Business Media B.V. 2010

\begin{abstract}
When modelling the turbulent dispersion of a passive tracer using Reynoldsaveraged Navier-Stokes (RANS) simulations, two different approaches can be used. The first consists of solving a transport equation for a scalar, where the governing parameters are the mean velocity field and the turbulent diffusion coefficient, given by the ratio of the turbulent viscosity and the turbulent Schmidt number $S c_{t}$. The second approach uses a Lagrangian particle tracking algorithm, where the governing parameters are the mean velocity and the fluctuating velocity field, which is determined from the turbulence kinetic energy and the Lagrangian time $T_{L}$. A comparison between the two approaches and wind-tunnel data for the dispersion in the wake of a rectangular building immersed in a neutral atmospheric boundary layer $(\mathrm{ABL})$ is presented. Particular attention was paid to the influence of turbulence model parameters on the flow and concentration field. In addition, an approach to estimate $S c_{t}$ and $T_{L}$ based on the calculated flow field is proposed. The results show that applying modified turbulence model constants to enable correct modelling of the ABL improves the prediction for the velocity and concentration fields when the modification is restricted to the region for which it was derived. The difference between simulated and measured concentrations is smaller than $25 \%$ or the uncertainty of the data on $76 \%$ of the points when solving the transport equation for a scalar with the proposed formulation for $S c_{t}$, and on $69 \%$ of the points when using the Lagrangian particle tracking with the proposed formulation for $T_{L}$.
\end{abstract}

Keywords Atmospheric boundary layer - Computational fluid dynamics · Dispersion · Lagrangian time scale $\cdot$ Numerical simulation · Turbulent Schmidt number

C. Gorlé

Department of Physics, EMAT, University of Antwerp, Groenenborgerlaan 171, 2020 Antwerp, Belgium e-mail: catherine.gorle@ua.ac.be

C. Gorlé $(\varangle)$ · J. van Beeck · P. Rambaud

von Karman Institute for Fluid Dynamics, Waterloosesteenweg 72, 1640 Sint-Genesius-Rode, Belgium e-mail: catherine.gorle@vki.ac.be 


\section{Introduction}

Computational fluid dynamics (CFD) modelling of pollutant dispersion in the lower part of the atmospheric boundary layer (ABL) has become an important research tool over recent years. Urban flow and dispersion have a highly turbulent character, and the correct modelling of the turbulent flow field and the resulting turbulent dispersion is a challenging task.

When modelling turbulent flows, one has the choice between using Reynolds-averaged Navier-Stokes (RANS) simulations and large-eddy simulations (LES). RANS simulations provide a time-averaged solution and require the modelling of the entire spectrum of turbulence scales. LES, on the other hand, provide an unsteady spatially filtered solution, where the larger, energy containing, turbulence scales are resolved and only the smaller scales need to be modelled. LES is inherently better suited for representing the intermittency of the urban dispersion process (Schatzmann and Leitl 2002), but its application is not a guarantee of a reliable solution. Specific care in the LES model set-up, such as the definition of the inflow boundary conditions and the wall boundary conditions, is required (Shah and Ferziger 1997; Lim et al. 2009). In addition, a well resolved LES requires a considerably higher computational power and time than RANS. Hence, RANS should still be considered a valuable tool when the aim is to calculate the time-averaged flow and concentration field, and further investigation of RANS modelling of the turbulent flow and dispersion phenomenon to improve the accuracy of the simulations is consequently worthwhile.

When considering RANS modelling of the dispersion of a passive tracer, two fundamentally different approaches can be distinguished: solving the flow equations in combination with a transport equation for a scalar, or solving the flow equations in combination with a particle tracking algorithm. In both cases, the dispersion is governed by both the mean velocity field and the turbulence field, which should therefore be resolved correctly in order to obtain a reliable solution for the concentration field. However, even after obtaining reliable velocity and turbulence fields, a considerable difference between the two approaches is introduced in the dispersion modelling, in particular with respect to the determination of the turbulent dispersion. When solving a transport equation for a scalar, the gradient-diffusion hypothesis is used and the turbulent diffusion coefficient is determined from the turbulent viscosity and the turbulent Schmidt number. When applying a particle tracking model, the fluctuating velocity component, determined from the turbulence kinetic energy and the Lagrangian time scale, is the governing parameter. Despite the fundamental difference between the two approaches, they should produce a comparable result when modelling the dispersion of particles with a sufficiently small relaxation time, which can be considered as a passive tracer.

In the present study the dispersion in the wake of a single, rectangular building has been modelled using RANS simulations with the standard $k-\varepsilon$ model, using both the standard production term and the Kato-Launder (KL) modification (Kato and Launder 1993), as implemented in the commercial CFD solver Fluent v6.3 (Fluent Inc. 2006). A comparison between concentration fields obtained from Lagrangian and Eulerian approaches has been performed. The Lagrangian approach employs a particle tracking algorithm, while the Eulerian approach solves the transport equation for a passive scalar. Wind-tunnel test data for the flow and concentration fields are available in datasets A1-1 and A1-5 from CEDVAL [compilation of experimental data for validation of microscale dispersion models, Leitl (1998)]. All simulations were performed at wind-tunnel scale (1:200) and the results were compared to the test data.

Considerable attention has been given to the correct modelling of the ABL, since it has been shown that both the velocity and turbulence profiles of the approach flow affect the results for the downstream velocity, turbulence and concentration (Castro and Robins 1977; 
Saathoff et al. 1998; Gao and Chow 2005). Two different approaches have been considered in this respect. The first one is based on a combination of the methods described by Blocken et al. (2007b) and Yang et al. (2007) and results in a streamwise homogeneous velocity field, but the turbulence field shows a streamwise variation through the domain. The second method includes an adjustment of constants in the turbulence model as presented in Gorlé et al. (2009), which reduces the streamwise variation of the turbulence field. A comparable approach was presented by Yang et al. (2009), while Yang et al. (2008) showed that modifying the turbulence model constants can improve the prediction of dynamic pressure on a low-rise building.

We present results for the flow around the rectangular building, modelled using both ABL modelling approaches to investigate the dependency of the solution on the values for the turbulence model constants. In addition, two hybrid modelling approaches were considered, in which the modified constants are only used in a part of the computational domain. The results for the velocity field and for the turbulence quantities of interest are compared to the test data for these quantities. When considering the dispersion in the wake of the building, the focus is on the determination of the remaining parameters that will influence the result for the concentration field: the Lagrangian time scale for the particle tracking algorithm and the turbulent Schmidt number for the transport equation for a passive scalar. Both parameters have been shown to affect the results for the concentration field (Tominaga and Stathopoulos 2007; Di Sabatino et al. 2007; Gorlé et al. 2009). In most previous studies, either the default values have been used, or the values were varied until an optimal agreement with the test data was obtained (Blocken et al. 2008; Nakiboglu et al. 2009). In the present study, an approach to estimate their values based on the solution obtained for the flow field is introduced. The results obtained using both methods are compared to each other and to the concentration field measurement data.

The following section presents the two approaches used for the ABL modelling and the respective results. In Sect. 3 the methodology used for the RANS CFD simulations is discussed, and the simulation results and a comparison with the test data are included in Sect. 4. Based on this comparison a discussion and conclusions are presented in Sects. 5 and 6.

\section{Atmospheric Boundary-Layer Model}

The boundary layer for CEDVAL A1-1 was modelled at a scale of 1:200 in the BLASIUS wind tunnel at the Meteorological Institute of the University of Hamburg (Donat 1995). Profiles of mean velocity and turbulence were obtained from two-component laser Doppler velocimetry measurements in one horizontal and one vertical plane. They are reported in terms of three mean velocity components $\langle U\rangle$ (longitudinal), $\langle V\rangle$ (lateral), $\langle W\rangle$ (vertical) $\left[\mathrm{m} \mathrm{s}^{-1}\right]$, three turbulence intensities $T I_{u}, T I_{v}, T I_{w}[\%]$ and two Reynolds stresses $\left\langle u^{\prime} v^{\prime}\right\rangle$ and $\left\langle u^{\prime} w^{\prime}\right\rangle$. For simulations with the $k-\varepsilon$ turbulence model, the boundary conditions for the turbulence should preferably be described in terms of the turbulence kinetic energy $k$ and the turbulence dissipation rate $\varepsilon$. In the present study the turbulence kinetic energy was determined from all three fluctuating velocity components: $\sqrt{\left\langle u^{\prime 2}\right\rangle}=T I_{u}\langle U\rangle, \sqrt{\left\langle v^{\prime 2}\right\rangle}=T I_{v}\langle U\rangle$, $\sqrt{\left\langle w^{\prime 2}\right\rangle}=T I_{w}\langle U\rangle$. The turbulence dissipation rate profile is obtained from the assumption of equilibrium between turbulence production and dissipation.

$$
k=\frac{1}{2}\left(\left\langle u^{\prime 2}\right\rangle+\left\langle v^{\prime 2}\right\rangle+\left\langle w^{\prime 2}\right\rangle\right),
$$


Table 1 Boundary conditions and model coefficients used for ABL modelling (the friction velocity $u_{*}$ and roughness length $z_{0}$ are obtained from fitting the velocity profile; the constants $A$ and $B$ from fitting the $k$ profile; the von Karman constant $\kappa$ equals 0.4187; the turbulence model constants $C_{1}$ and $C_{2}$ are 1.44 and 1.92 respectively; $z_{1}$ is the wall-normal coordinate of the centre point of the wall adjacent cell)

\begin{tabular}{lll}
\hline Inlet profiles & $\langle U\rangle$ & $\frac{u_{*}}{\kappa} \ln \frac{z+z_{0}}{z_{0}}$ \\
& $k$ & $\sqrt{A \ln \left(z+z_{0}\right)+B}$ \\
Wall function & $\varepsilon$ & $\frac{\sqrt{C_{\mu}} u_{*}}{\kappa\left(z+z_{0}\right)} \sqrt{A \ln \left(z+z_{0}\right)+B}$ \\
Coefficients & $k_{S}$ & $\leq z_{1}$ \\
Turbulence model coefficient & $C_{S}$ & $\frac{9.793 z_{0}}{k_{s}}$ \\
Turbulence dissipation Prandtl number & $C_{\mu}$ & $\frac{u_{*}^{4}}{A \ln \left(z_{1}+z_{0}\right)+B}$ \\
& $\sigma_{\varepsilon}$ & $\frac{-A / 2+k(z)^{2}}{\left(C_{2}-C_{1}\right) \frac{u_{*}^{2}}{\kappa^{2}} k(z)}$ \\
\hline
\end{tabular}

$$
\varepsilon=\sqrt{C_{\mu}} k(z) \frac{d\langle U\rangle}{d z},
$$

where $C_{\mu}$ is the turbulence model constant relating the turbulent viscosity $\mu_{t}$ to the turbulence kinetic energy and dissipation rate: $\mu_{t}=\rho C_{\mu} k^{2} / \varepsilon$.

When using fully developed ABL profiles as an inlet condition for CFD simulations with the standard wall functions and model coefficients, these profiles will not be maintained throughout the computational domain (Riddle et al. 2004; Franke et al. 2004, 2007; Blocken et al. 2007a, b; Hargreaves and Wright 2007). The reason for streamwise variations in the profiles is an incompatibility of the inlet profiles with the applied wall functions and the model equations. Several solutions for this problem have been proposed. In the present study, the methodology established in Gorlé et al. (2009) was used, which provides an approximate solution to the system of equations describing a fully developed ABL with the standard $k-\varepsilon$ model. The method is based on the approaches described by Richards and Hoxey (1993); Blocken et al. (2007b) and Yang et al. (2007) and provides good streamwise homogeneity for both the velocity and the turbulence profiles. It requires the specification of inlet profiles, wall-function roughness height $k_{s}$ and roughness constant $C_{s}$, and turbulence model constants $C_{\mu}$ and $\sigma_{\varepsilon}$ (the turbulence dissipation Prandtl number) as included in Table 1.

A reasonable fit to the profiles from the wind-tunnel test was obtained using $u_{*}=0.374$ $\mathrm{m} \mathrm{s}^{-1}, \kappa=0.41, z_{0}=0.00075 \mathrm{~m}, A=0.025$ and $B=0.41 . k_{s}$ was equal to the wall adjacent cell centre height $(0.00425 \mathrm{~m})$, implying a value of 1.73 for $C_{s}$. The calculated value for $C_{\mu}$ was 0.07 , as opposed to the standard value of 0.09 . The value for $\sigma_{\varepsilon}$ varied from 1.35 in the wall adjacent cell to 1.62 at the top boundary, as opposed to the standard value of 1.3.

Figure 1 shows the influence of modifying the constants on the streamwise homogeneity of the velocity, the turbulence kinetic energy and the turbulent viscosity of the ABL when performing two-dimensional simulations. The computational domain was $4 \mathrm{~m}$ long and $1 \mathrm{~m}$ high, the structured quadrilateral grid consisted of $400 \times 70$ cells with the centre of the wall adjacent cell at a height of $0.00425 \mathrm{~m}\left(z^{+} \approx 120\right)$. The standard $k-\varepsilon$ model was used and all discretisation schemes were second-order upwind. It is shown that adjusting the constants results in an improved streamwise homogeneity of the turbulence kinetic energy profile. For the turbulent viscosity $\mu_{t}$, the values at the domain inlet also depend on the constant $C_{\mu}$. Adjusting the constant results in better agreement with the value obtained from the test data, which was determined using $\mu_{t}=-\rho\left\langle u^{\prime} w^{\prime}\right\rangle /\left[u_{*} / \kappa\left(z+z_{0}\right)\right]$, according to the Boussinesq hypothesis combined with the logarithmic velocity profile. 

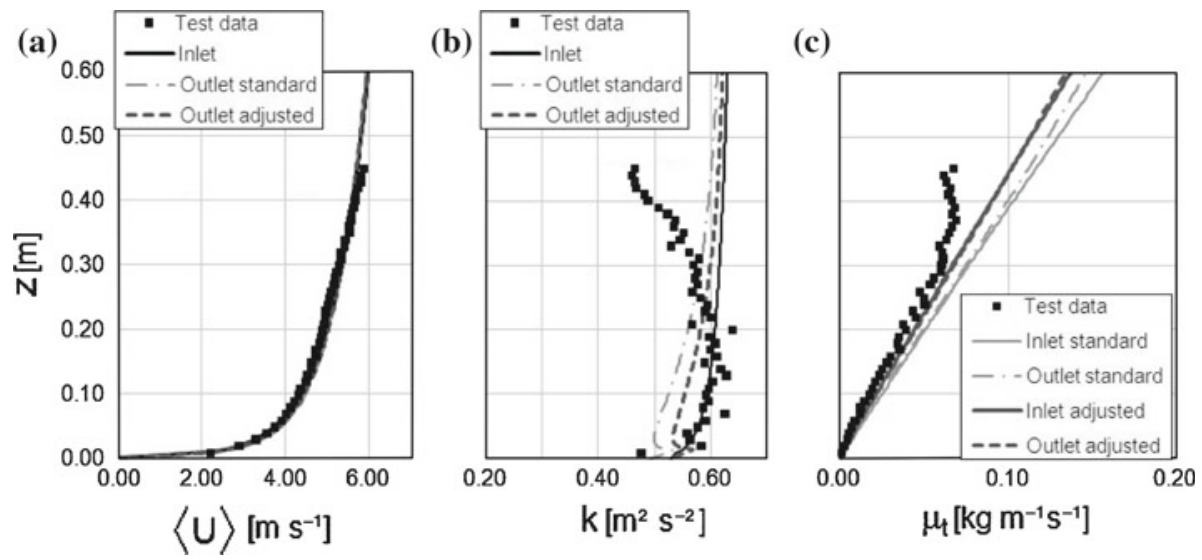

Fig. 1 ABL profiles from measurements and from CFD at inlet and outlet of simulation domain: mean streamwise velocity component (a), turbulence kinetic energy (b) and turbulent viscosity (c)

The influence of changing the turbulence model constants $C_{\mu}$ and $\sigma_{\varepsilon}$ on the simulations of the flow and dispersion around a rectangular building should now be verified. This is done by comparing simulations with the standard and the adjusted constants as explained in the next section.

\section{Modelling Approaches for Dispersion in the Wake of a Rectangular Building}

\subsection{Computational and Physical Model for the Flow}

Figure 2 shows the building geometry, which has length $L=0.1 \mathrm{~m}$, width $W=0.15 \mathrm{~m}$ and height $H=0.125 \mathrm{~m}$ and four source elements on the leeward side from which the tracer gas is introduced. The inlet of the computational domain is defined $1 \mathrm{~m}$ upstream of the building, since the measurement of the inlet ABL profiles was executed at this location in the wind tunnel. The outlet is located $4 \mathrm{~m}$ downstream of the building, which is larger than the minimum recommended longitudinal domain extension (Franke et al. 2007). The width and height of the domain are $0.65 \mathrm{~m}$ and $1 \mathrm{~m}$ respectively, corresponding to the wind-tunnel dimensions.
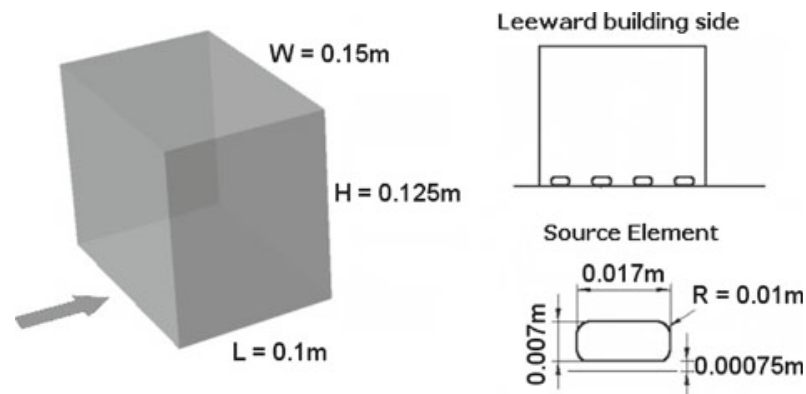

Fig. 2 Building geometry 
As the model is symmetrical with respect to the plane $y=0$, only half of the domain was numerically represented. A simulation representing the complete domain was initially performed, and converged to an identical steady-state solution.

The computational grid consists of 1.3 million cells. The (half) building is discretised using $20 \times 26 \times 40$ elements, while each source element contains $6 \times 4$ cells. The height of the ground adjacent cell near the building is $0.00075 \mathrm{~m}\left(z^{+} \approx 10\right)$, resulting in one cell between the ground surface and the lower edge of the source elements. In the region further away from the building ( $1 H$ upstream, $1 H$ to the side and $4 H$ downstream), the height of the first cell centre is at $0.00425 \mathrm{~m}\left(z^{+} \approx 120\right)$ to allow a correct modelling of the ABL as discussed in Sect. 2. Except for the first cell, all nodes at the interfaces are conformal. A grid dependency study was performed by solving the flow and dispersion on a grid refined by a factor of 2 in each direction within the vicinity of the building and the differences observed in the velocity at the measurements points were on average smaller than $0.5 \%$ of the reference velocity and a maximum of $5 \%$ of the reference velocity.

The boundary conditions at the inlet are as described in Sect. 2. The source elements are velocity inlets from which air is introduced in the flow field with a velocity of $0.024 \mathrm{~m} \mathrm{~s}^{-1}$. The resulting total source flow rate $Q_{s}$ is $1.14 \times 10^{-5} \mathrm{~m}^{3} \mathrm{~s}^{-1}$. When using the Lagrangian particle tracking algorithm particles are released from random locations within the source elements with the same velocity, while for the Eulerian model the user-defined scalar value at the source elements is fixed to 1 . At the outlet a zero static pressure is defined. On the side and upper boundaries, a smooth-wall boundary condition is imposed and the plane $y=0$ is a symmetry plane. At the ground, the roughness length $k_{s}$ and constant $C_{s}$ are as defined in Sect. 2 in the region where the first cell height is $0.00425 \mathrm{~m}$. Close to the building area, where the first cell height is smaller, the standard logarithmic law-of-the-wall for smooth walls is applied, or, where $z^{+}$is smaller than about 10, the laminar stress-strain relationship is used.

Turbulence is modelled using the standard $k-\varepsilon$ model. Simulations with the standard $k-\varepsilon$ model are known to overpredict the production of turbulence in the stagnation region, and so simulations were also performed with the Kato-Launder (KL) modification for the production term of the turbulence kinetic energy (Kato and Launder 1993). In the standard $k-\varepsilon$ model, the turbulent production is given by $P_{k}=2 \mu_{t} S_{i j} S_{i j}$, where $S_{i j}$ is the mean strain rate defined as $S_{i j}=\frac{1}{2}\left(\frac{\partial\left\langle U_{i}\right\rangle}{\partial x_{j}}+\frac{\partial\left\langle U_{j}\right\rangle}{\partial x_{i}}\right)$. The turbulence production determined from this relation is always positive. Kato and Launder suggested an alternative formulation for the production, $P_{k}=2 \mu_{t} S_{i j} \Omega_{i j}$, where $\Omega_{i j}$ is the mean rate of rotation given by $\Omega_{i j}=\frac{1}{2}\left(\frac{\partial\left\langle U_{i}\right\rangle}{\partial x_{j}}-\frac{\partial\left\langle U_{j}\right\rangle}{\partial x_{i}}\right)$. The production in the stagnation point will be negligible using this expression, since the flow is nearly irrotational at this location. It has been shown by Lakehal and Rodi (1997) that the resulting solution for the turbulence kinetic energy field when considering flows over rectangular buildings improves significantly.

In Sect. 2 it was introduced that the streamwise homogeneity of the turbulence kinetic energy profile defined at the inlet can be improved by adjusting the turbulence model constants $C_{\mu}$ and $\sigma_{\varepsilon}$ according to the formulations from Table 1 . These equations were derived for an undisturbed ABL with equilibrium between turbulence production and dissipation, which is not valid in the wake or within the influence area of the building. Therefore, the influence of modifying these constants when solving the flow around a building was investigated by performing four different simulations using identical inlet and wall boundary conditions, but varying the two constants as indicated in Table 2 . 
Table 2 Settings of turbulence model constants considered in simulations

$\begin{aligned} & k-\varepsilon \text { Standard } \\ & k-\varepsilon \text { Modified }\end{aligned}$
$\begin{aligned} & \text { Standard constants in entire computational domain } \\ & \text { Modified constants in entire computational domain }\end{aligned}$
Modified constants, but standard constants in the wake (region
extending 2 building heights downstream)
Modified constants, but standard constants within the influence area of the
building [half sphere, $r=1.76 H$, centre $0.16 H$ downstream of building
Beranek (1979)]

\subsection{Dispersion Modelling Using a Lagrangian Particle Tracking Algorithm}

\subsubsection{Model Description}

The particle tracking algorithm used is the Discrete Phase Model (DPM) implemented in Fluent v6.3 (Fluent Inc. 2006) with one-way coupling, which determines the particle trajectory by integrating the force balance of the particle written in a Lagrangian reference frame. In the present analysis the only force considered is the drag force, hence the force balance can be written as:

$$
\frac{d \mathbf{U}_{\mathbf{p}}}{d t}=\frac{18 \mu}{d_{p}^{2} \rho_{p} C_{c}}\left(\mathbf{U}-\mathbf{U}_{\mathbf{p}}\right),
$$

where $\mathbf{U}$ is the instantaneous velocity vector, $\mathbf{U}_{\mathbf{p}}$ is the instantaneous particle velocity vector, $d_{p}$ is the particle diameter, $\rho_{p}$ is the particle density and $C_{c}$ is the Cunningham correction factor. The factor $\frac{\rho_{p} d_{p}^{2} C_{c}}{18 \mu}$ is the particle relaxation time $\tau_{p}$, which equals $3 \times 10^{-6} \mathrm{~s}$. Particles with such a small relaxation time respond quickly to changes in the carrier flow field and behave as a passive tracer.

The turbulent dispersion is accounted for by integrating the trajectory equations for individual particles using the instantaneous fluid velocity $\mathbf{U}=\langle\mathbf{U}\rangle+\mathbf{u}^{\prime}(\mathbf{t})$ along the particle path. The instantaneous fluid velocity is determined using a stochastic method, i.e. the random walk model (Fluent Inc. 2006), in which the fluctuating velocity components are assumed to be isotropic and determined as discrete piecewise constant functions of time given by $u^{\prime}=v^{\prime}=w^{\prime}=\zeta \sqrt{2 k / 3}$. Here, $\zeta$ is a normally distributed random number between -1 and 1 , kept constant over an interval of time that is representative of the time that a particle is assumed to interact with an eddy. This value is given by the smaller value of the characteristic lifetime of the eddies and the particle eddy crossing time. The particle eddy crossing time is defined as $t_{\text {cross }}=-\tau_{p} \ln \left[1-L_{e} /\left(\tau_{p}\left|\mathbf{U}-\mathbf{U}_{\mathbf{p}}\right|\right)\right]$ where $\tau_{p}$ is the particle relaxation time and $L_{e}$ is the eddy length scale. The characteristic life time is given by $\tau_{e}=-T_{L} \log (r)$ where $r$ is a random number between 0 and 1 and $T_{L}$ is the Lagrangian time scale, calculated from:

$$
T_{L} \approx C \frac{k}{\varepsilon}
$$

The value for the constant $C$ can influence the results of the particle tracking algorithm considerably (Gorlé et al. 2009). Since there is no universal value that is valid for all flow problems, the following section describes a method to determine the constant based on the solution obtained for the flow field. 


\subsubsection{Determining $C$ for Calculation of $T_{L}$ from a Solution for the Flow Field}

In homogeneous, isotropic and stationary turbulence, according to Oesterlé (2006), the value for $C$ in Eq. 4 can be determined from:

$$
C=\frac{4}{3 C_{0}}
$$

with

$$
C_{0}=\frac{C_{0 \infty}}{1+7.5 C_{0 \infty}^{2} R e_{\lambda}^{-1.64}} .
$$

In this equation $C_{0 \infty}$ is approximately equal to 6 , and $R e_{\lambda}$, the Taylor-scale Reynolds number, was determined from (Pope 2008):

$$
R e_{\lambda}=\sqrt{\frac{20}{3} R e_{L}}=\sqrt{\frac{20}{3} \frac{k^{2}}{\varepsilon v}} .
$$

Using these equations a field for $C$ can be extracted. The final value used for the determination of the Lagrangian time scale was determined by taking the volume average of the values obtained within the region of interest. The resulting value for $C$ was 0.23 or 0.24 , depending on the simulation settings, compared to the default value of 0.15 .

Because of the assumption of homogeneous, isotropic and stationary turbulence, the values obtained for $C$ might not be accurate in regions where these assumptions are not valid. This is especially the case in regions where the flow is strongly inhomogeneous, e.g. near the building corners, and in regions where the turbulence is anisotropic. The validity of the relation in the vicinity of the building is consequently a subject of further research as discussed in Sect. 6. It is noted, however, that the commonly applied strategy of assuming the default value for the constant $C$, which cannot account for the specific characteristics of the flow field in any way, is likely to be less accurate.

\subsubsection{Converting Particle Paths to Concentration Values}

Concentrations at specified points inside the domain are determined by calculating the time that each particle spends inside a small cell volume surrounding the point of interest. By summing this time over the total number of particles tracked, $N_{p}$, the total time spent by all particles in the cell, $\sum t_{i, \text { cell }}$, can be determined. The non-dimensional concentration $C^{*}=C_{\text {local }} / C_{\text {source }}$ is then given by:

$$
C_{\text {cell }}^{*}=\frac{Q_{s} \sum t_{i, \text { cell }}}{N_{p} V_{\text {cell }}}
$$

where $Q_{s}$ is the source strength (source flow rate) and $V_{\text {cell }}$ is the volume of the cell. A sufficiently large number of particles should be tracked to obtain concentration values with an acceptable level of uncertainty. In the present simulations, the concentration was determined by releasing 80,000 particles from the two source elements represented in the model. For the simulations with the standard $k-\varepsilon$ model this procedure was repeated five times to allow the calculation of an average and a standard deviation, from which the uncertainty on the concentration values could be estimated. The results of this analysis are summarised in the Appendix. 
3.3 Dispersion Modelling by Solving the Transport Equation for a Passive Scalar

\subsubsection{Model Description}

The transport equation for a passive scalar was solved using Fluent's User-Defined-Scalar (UDS) capability (Fluent Inc. 2006). The time-averaged transport equation for an arbitrary scalar $\phi$ in steady conditions is given by:

$$
\frac{\partial}{\partial x_{i}}\left[\left\langle U_{i}\right\rangle\langle\phi\rangle-D \frac{\partial\langle\phi\rangle}{\partial x_{i}}\right]=\left\langle S_{\phi}\right\rangle
$$

where $\left\langle S_{\phi}\right\rangle$ is a time-averaged source term and $D$ is the diffusion coefficient, which is given by the sum of the molecular mass diffusion coefficient $D_{m}$ and the turbulent mass diffusion coefficient $D_{t}$. The turbulent diffusion coefficient is usually defined as:

$$
D_{t}=\frac{v_{t}}{S c_{t}}
$$

where $v_{t}$ is the turbulent kinematic viscosity and $S c_{t}$ is the turbulent Schmidt number. The value of $S c_{t}$ significantly influences the results for the concentration field (Tominaga and Stathopoulos 2007). The reported optimum values for $S c_{t}$ in the literature are widely distributed in the range of $0.2-1.3$. Since there is no universal value for $S c_{t}$ that is valid for all flow problems, the following section describes a method to estimate its value based on the solution obtained for the flow field.

\subsubsection{Determining $S c_{t}$ from a Solution for the Flow Field}

The turbulent diffusion coefficient in homogeneous, stationary and isotropic turbulence can be determined from (Oesterlé 2006):

$$
D_{t}=\frac{1}{2} C_{0} \varepsilon T_{L}^{2}
$$

Substituting Eqs. 4 and 5 one obtains:

$$
D_{t}=\frac{8}{9 C_{0}} \frac{k^{2}}{\varepsilon},
$$

with the constant $C_{0}$ as defined in Eq. 6. Combining this with Eq. 10, and with the definition of the turbulent kinematic viscosity $v_{t}=C_{\mu} k^{2} / \varepsilon$, one obtains:

$$
S c_{t}=\frac{9}{8} C_{\mu} C_{0} .
$$

Using this expression in combination with Eq. 6 a field for $S c_{t}$ can be extracted. The final value used for the determination of the turbulent diffusion coefficient was determined by taking the volume average of the values obtained within the region of interest. The resulting value for $S c_{t}$ depended on the settings for the turbulence model constants (since these affect the value for $v_{t}$ ) and varied between 0.42 and 0.54 . These values are within the range of some recent studies comparing CFD with proven integral models and experiments for dispersion in the ABL (Riddle et al. 2004; Di Sabatino et al. 2007; Blocken et al. 2008; Nakiboglu et al. 2009), which showed that reducing $S c_{t}$ from the default value of 0.7 to values around 0.4 improves the predicted concentration levels. 
As for the determination of the constant $C$ used for calculating the Lagrangian time scale, the formulation for $S c_{t}$ assumes homogeneous, isotropic and stationary turbulence. The validity of the relation in the vicinity of the building, where the flow can be strongly inhomogeneous and anisotropic, is consequently a subject of further research as discussed in Sect. 6. It is noted, however, that the commonly applied strategy of assuming the default value for the turbulent Schmidt number, which cannot account for the specific characteristics of the flow field in any way, is likely to be less accurate.

\subsection{Solution Procedure}

The Fluent v6.3 steady, three-dimensional, double precision, pressure-based solver was used. The discretisation scheme for the pressure was set to standard, for the momentum and turbulence quantities the second-order upwind schemes were used, and for the pressure-velocity coupling the SIMPLE scheme was applied. The simulations were run until the residuals levelled out, which resulted in a decrease in their value of at least six orders of magnitude. In addition the solution was monitored to ensure that the flow variables converged to a stable solution.

\section{Comparison of Results with Test Data}

The CFD results were post-processed by extracting data on the available measurement points, which form sets of planes as indicated in Fig. 3.

The results for the flow field are compared by plotting contours of the time-averaged streamwise non-dimensional velocity component, $\langle U\rangle / U_{\text {ref }}$, and the non-dimensional turbulence kinetic energy, $k / U_{\text {ref }}^{2}$ on the planes $y=0$ and $z=0.035 \mathrm{~m} ; U_{\text {ref }}$ is the streamwise reference velocity.

Concentration measurements are available on all planes shown on the right of Fig. 3. The concentration is expressed non-dimensionally as:

$$
K=\frac{C_{l}}{C_{s}} \frac{U_{\mathrm{ref}} H^{2}}{Q_{s}},
$$

where $C_{l}$ is the local concentration, $C_{s}$ is the concentration at the source, and $Q_{s}$ is the source strength (source flow rate). Contour plots of $K$ on the planes $y=0.076 \mathrm{~m}$ and $z=0.035 \mathrm{~m}$ are presented.
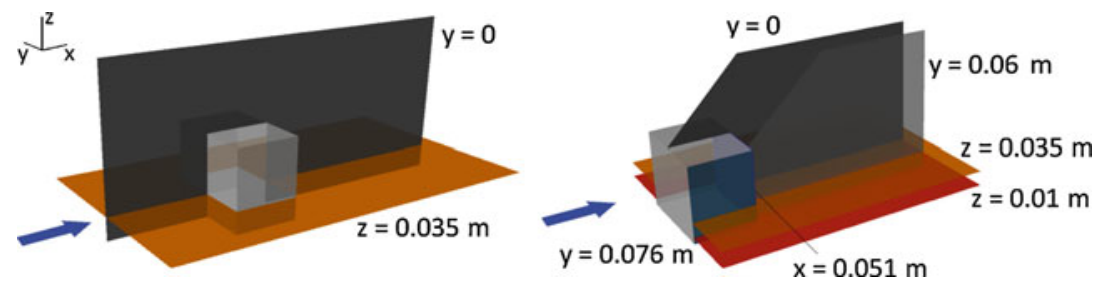

Fig. 3 Location of measurement points for the flow variables (left) and the concentration (right) 
To quantify the results, a hit rate $q$ was determined for all three quantities as follows:

$$
q=\frac{1}{N} \sum_{i=1}^{N} \delta_{i}
$$

with

$$
\delta_{i}=\begin{array}{ll}
1 & \text { for } \\
0 & \text { else. }
\end{array}\left|\frac{V_{\mathrm{CFD}}-V_{\text {Test }}}{V_{\text {Test }}}\right| \leq 0.25 \text { or }\left|V_{\mathrm{CFD}}-V_{\text {Test }}\right| \leq W
$$

The hit rate indicates the fraction of $N$ measurement points at which the CFD results are within $25 \%$ of the measurements or within the uncertainty interval $W$ of the data (see Appendix). The value used for the uncertainty affects the absolute value of the hit rate, but was verified not to change the relative model performance. Both the total hit rate for all measurement points, and local hit rates for selected points upstream of the building, to the side and on top of the building, and downstream of the building, are presented.

\subsection{Comparison of the Results for the Flow Field}

Contour plots of $\langle U\rangle / U_{\text {ref }}$ and $k / U_{\text {ref }}^{2}$ on the symmetry plane are shown in Figs. 4 and 5. When considering the velocity field it is obvious that the main influence of varying the turbulence model constants or the production term is found in the predicted wake length, $L_{w}$, which is indicated in the plots. For the turbulence field, the main differences are found in the stagnation region and in the building wake.

The standard model overpredicts the wake length. Applying the modified constants in the entire computational domain increases the wake length further as a result of a small decrease of the turbulence kinetic energy to the sides of the building, combined with a slightly larger separation bubble at this location. This effect is eliminated when applying the standard constants in the wake (hybrid 1), which also induces an increase in the turbulence kinetic energy in the wake. The best agreement in the wake length is found using the hybrid 2 settings, where the decreased size of the separation bubble on the building side significantly decreases the wake length. In this case, however, there is a large overprediction of the turbulence kinetic energy in all regions. Except for the hybrid 2 case, the KL correction results in an increased wake length, as a result of the decreased turbulence production in front of the building.

In summary, and as confirmed by the values for the hit rate presented in Table 3, the hybrid 2 settings with the KL correction produce the best result for the velocity field, while the hybrid 1 settings with the KL correction perform better when considering the turbulence kinetic energy. The dispersion results depend both on the mean velocity and the turbulence field, so a trade-off between a good prediction of the velocity field and the wake length and a good prediction of the turbulence field is required. In order to investigate which is the more important, the flow results for the $k-\varepsilon$ standard, the $k-\varepsilon$ hybrid $1, k-\varepsilon$ hybrid 1 with KL, $k-\varepsilon$ hybrid 2 and $k-\varepsilon$ hybrid 2 with KL were used for calculating the concentration field.

\subsection{Comparison of the Results for the Concentration Field}

Figures 6 and 7 present the contours of the non-dimensional concentration $K$ on the planes $y=0.076 \mathrm{~m}$ and $z=0.035 \mathrm{~m}$. The main differences between the concentration patterns are observed in the upstream pollutant dispersion to the sides of the building and in the downstream concentration pattern. To quantify these observations Table 4 presents the hit rate for 

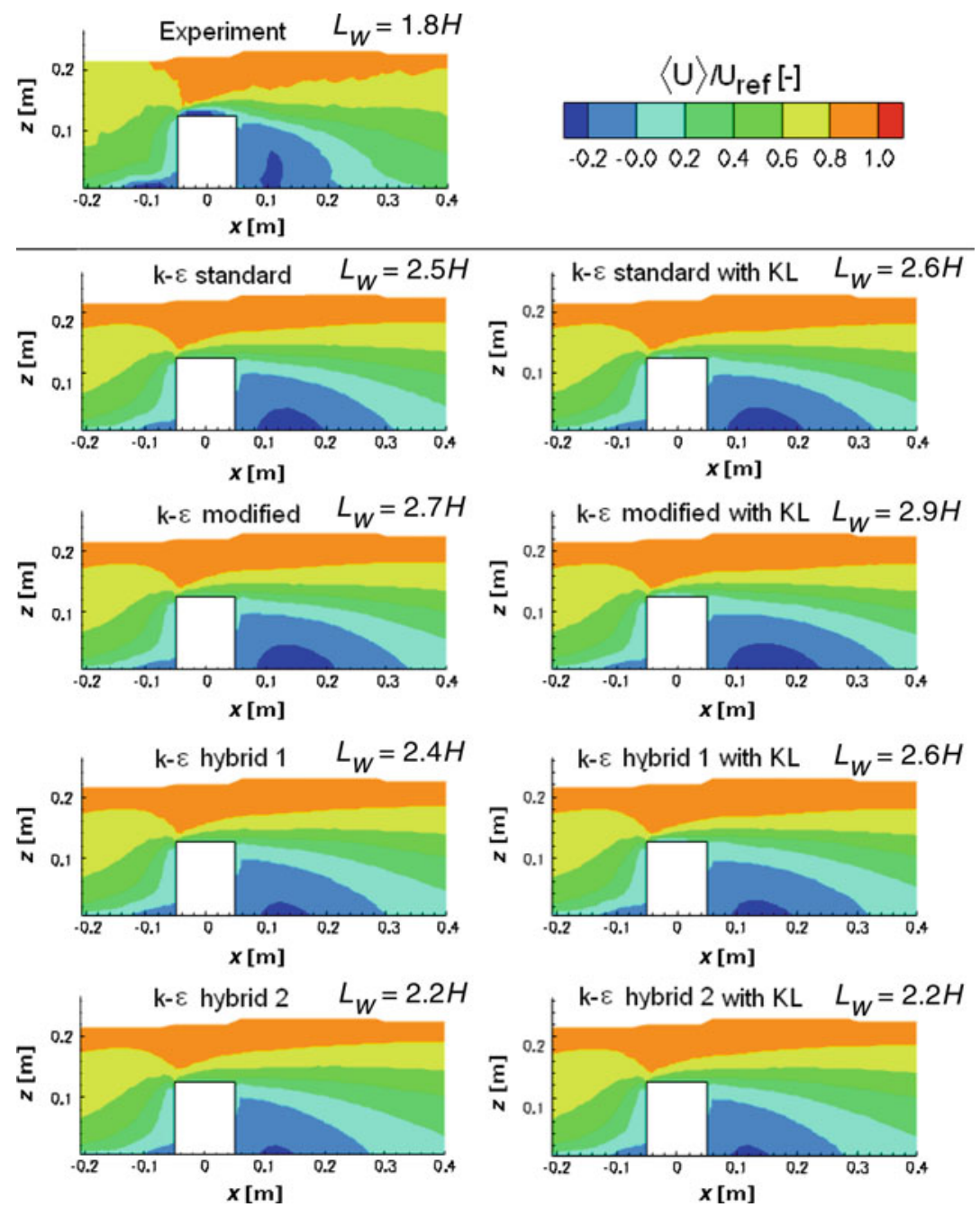

Fig. 4 Contours of the non-dimensional streamwise velocity component

all measurement points upstream of the source to the side of the building ( $y=0.076 \mathrm{~m}$ and selected points on $z=0.01 \mathrm{~m}$ and $z=0.035 \mathrm{~m}$ ) and for the measurement points downstream of the source (on $y=0.06 \mathrm{~m}$ and selected points on $y=0, z=0.01 \mathrm{~m}$ and $z=0.035 \mathrm{~m}$ ). In addition the hit rate over all points is presented.

Both from the hit rate and from Fig. 6 it can be observed that the UDS model with the hybrid 1 settings and the KL correction shows the best performance to the side of the building. The standard and hybrid 1 settings in combination with the UDS model show a more severe underprediction around half the building height. The standard and both hybrid 1 settings in combination with the DPM model significantly overpredict the concentration close to the ground, which is reflected in lower values for the hit rate than obtained with the UDS model. When applying the hybrid 2 settings, the decrease of the separation bubble size on the building side results in a significant underprediction of the upstream pollutant dispersion for both models. This can also be seen in Fig. 7. 

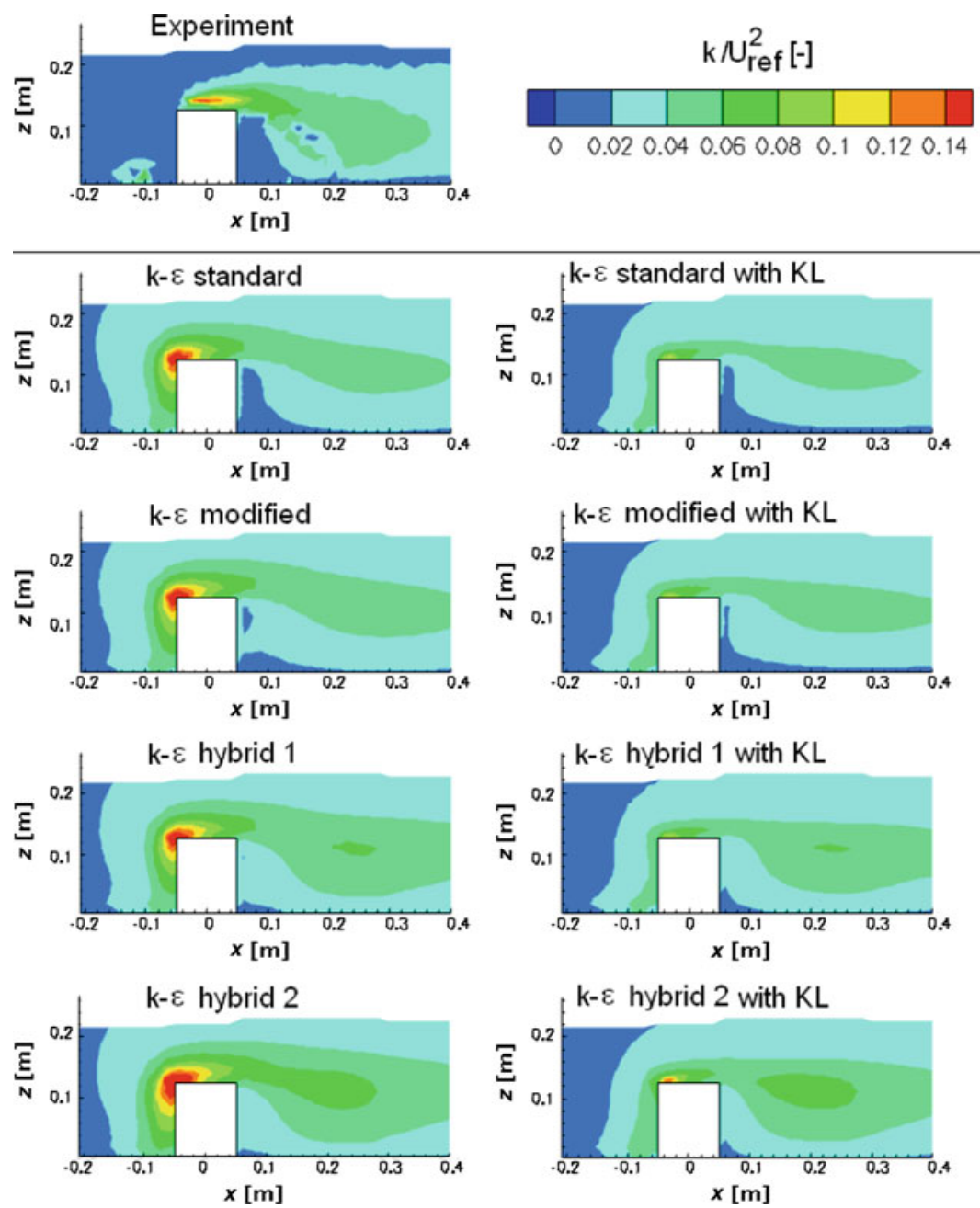

Fig. 5 Contours of the non-dimensional turbulence kinetic energy

Table 3 Hit rate for $\langle U\rangle / U_{\text {ref }}$ and $k / U_{\text {ref }}^{2}$

\begin{tabular}{|c|c|c|c|c|c|c|c|c|}
\hline & \multicolumn{4}{|l|}{$\langle U\rangle / U_{\mathrm{ref}}$} & \multicolumn{4}{|l|}{$k / U_{\mathrm{ref}}^{2}$} \\
\hline & Upstream & Side \& top & Downstream & All & Upstream & Side \& top & Downstream & All \\
\hline$k-\varepsilon$ Standard & 0.89 & 0.76 & 0.56 & 0.69 & 0.47 & 0.38 & 0.65 & 0.55 \\
\hline$k-\varepsilon$ Standard KL & 0.88 & 0.75 & 0.54 & 0.68 & 0.53 & 0.45 & 0.67 & 0.59 \\
\hline$k-\varepsilon$ Modified & 0.88 & 0.75 & 0.54 & 0.68 & 0.51 & 0.34 & 0.61 & 0.54 \\
\hline$k-\varepsilon$ Modified KL & 0.88 & 0.74 & 0.50 & 0.66 & 0.56 & 0.45 & 0.67 & 0.60 \\
\hline$k-\varepsilon$ Hybrid 1 & 0.89 & 0.77 & 0.55 & 0.69 & 0.51 & 0.35 & 0.59 & 0.53 \\
\hline$k-\varepsilon$ Hybrid $1 \mathrm{KL}$ & 0.89 & 0.75 & 0.52 & 0.67 & 0.55 & 0.45 & 0.68 & 0.60 \\
\hline$k-\varepsilon$ Hybrid 2 & 0.87 & 0.79 & 0.57 & 0.70 & 0.44 & 0.22 & 0.45 & 0.41 \\
\hline$k-\varepsilon$ Hybrid $2 \mathrm{KL}$ & 0.87 & 0.81 & 0.59 & 0.71 & 0.54 & 0.31 & 0.49 & 0.47 \\
\hline
\end{tabular}




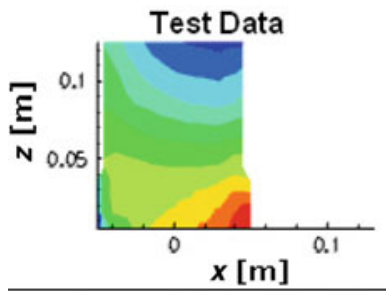

$\mathrm{K}[-]$
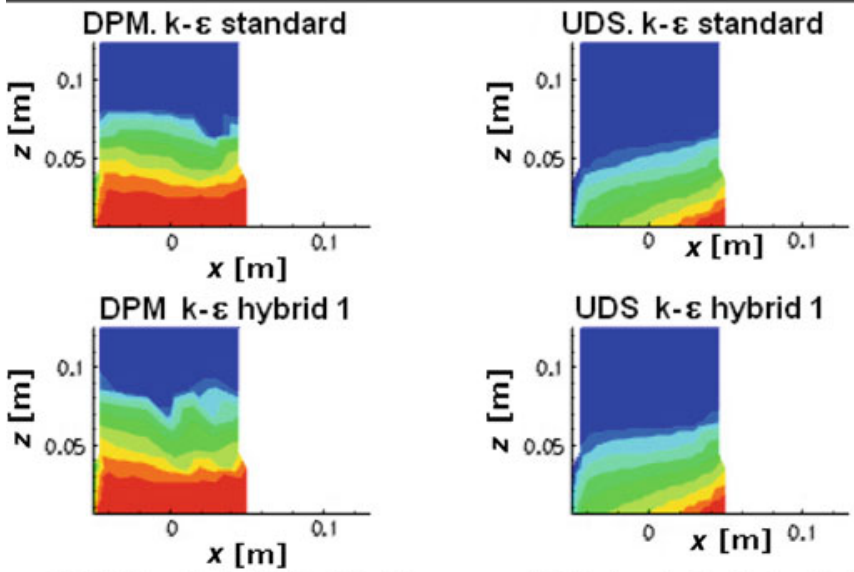

DPM k- $\varepsilon$ hybrid 1 with $\mathrm{KL}$

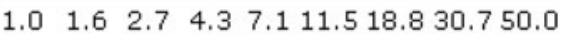
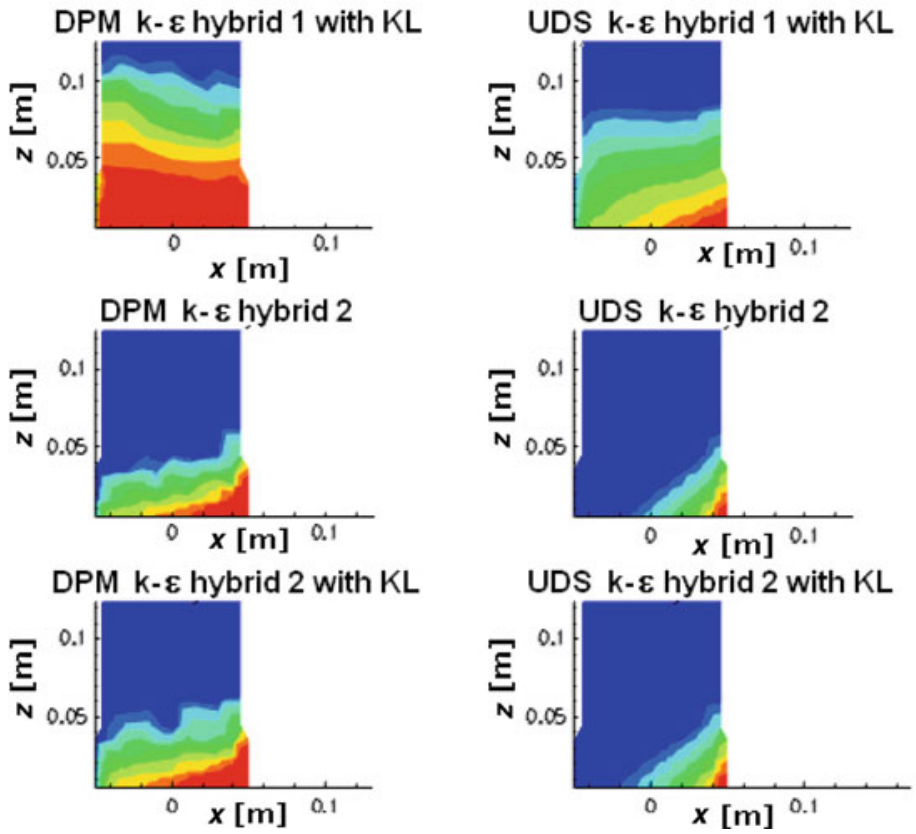

Fig. 6 Contours of non-dimensional concentration on the plane $y=0.076 \mathrm{~m}$

Downstream of the source a relatively high hit rate is obtained for all models. This is related to the fact that this region contains a high percentage of low concentration measurement points, thereby increasing the allowed relative discrepancy for which a hit is obtained (see Appendix). The hybrid 2 settings show the lowest hit rate in the downstream region. 

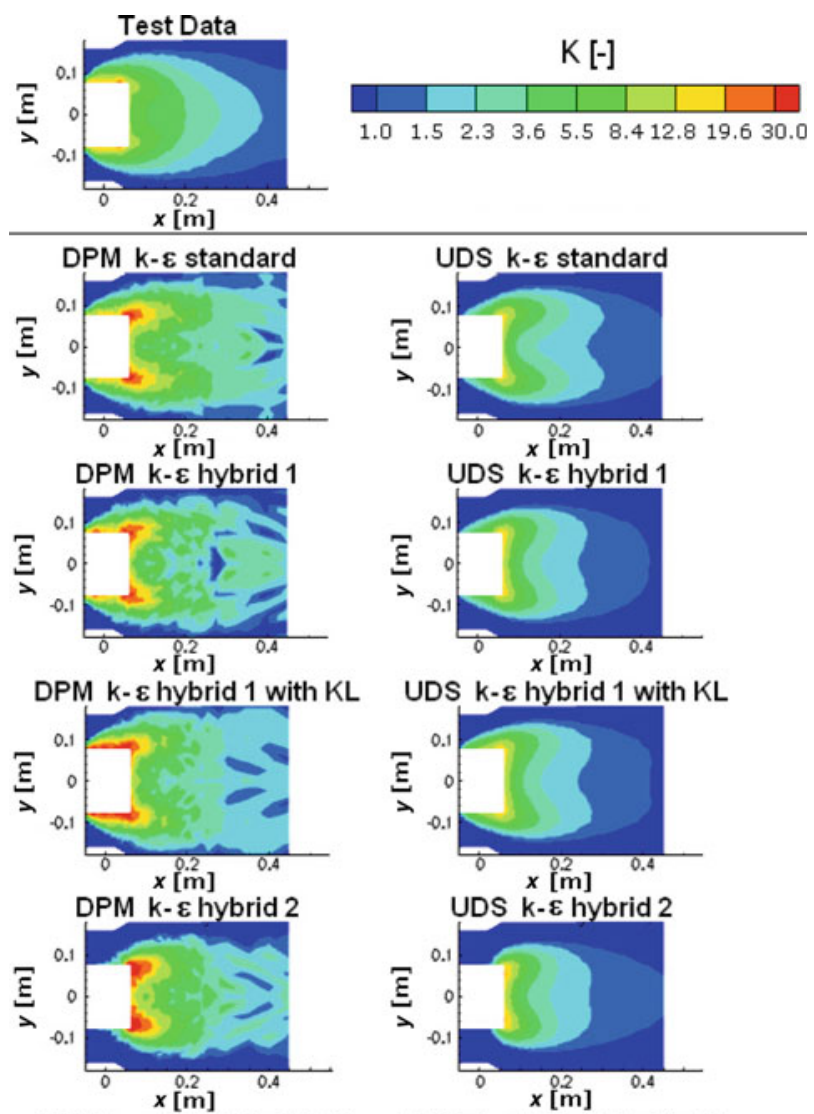

DPM k- $\varepsilon$ hybrid 2 with $\mathrm{KL}$

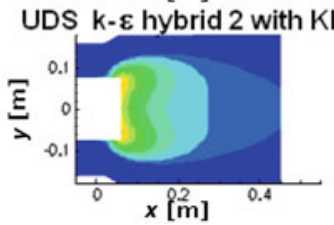

Fig. 7 Contours of non-dimensional concentration on the plane $z=0.035 \mathrm{~m}$

The reason for this can be observed in Fig. 7 and is twofold: the upwards dispersion of the tracer just downstream of the source is overpredicted (mainly for the DPM model), while the concentrations further downstream are underpredicted.

Overall, it is observed that the UDS model performs slightly better than the DPM model. For both models, the performance of the standard and the hybrid 1 models is better than that of the hybrid 2 settings. The UDS model with the hybrid 1 settings and the KL modification shows the best overall performance.

\section{Discussion}

An interesting observation is that the simulations for the hybrid 2 case, which provide the best prediction of the wake length, produce the worst prediction for the concentration field. 
Table 4 Hit rate for non-dimensional concentration $K$

\begin{tabular}{llll}
\hline & $\begin{array}{l}\text { Upstream } \\
\text { of source }\end{array}$ & $\begin{array}{l}\text { Downstream } \\
\text { of source }\end{array}$ & All \\
\hline DPM, $k-\varepsilon$ Standard & 0.54 & 0.77 & 0.68 \\
DPM, $k-\varepsilon$ Hybrid 1 & 0.51 & 0.81 & 0.70 \\
DPM, $k-\varepsilon$ Hybrid 1 with KL & 0.44 & 0.82 & 0.69 \\
DPM, $k-\varepsilon$ Hybrid 2 & 0.36 & 0.70 & 0.58 \\
DPM, $k-\varepsilon$ Hybrid 2 with KL & 0.39 & 0.71 & 0.60 \\
UDS, $k-\varepsilon$ Standard & 0.57 & 0.81 & 0.71 \\
UDS, $k-\varepsilon$ Hybrid 1 & 0.58 & 0.82 & 0.72 \\
UDS, $k-\varepsilon$ Hybrid 1 with KL & 0.66 & 0.83 & 0.76 \\
UDS, $k-\varepsilon$ Hybrid 2 & 0.44 & 0.78 & 0.62 \\
UDS, $k-\varepsilon$ Hybrid 2 with KL & 0.43 & 0.77 & 0.62 \\
\hline
\end{tabular}

Two main differences are observed. First, the downstream concentrations are overpredicted close to the source and underpredicted further downstream. Second, the concentrations to the side of the building are underpredicted. Both observations indicate that the pollutant remains concentrated near the source and is less dispersed than in the experiment, which seems to be in contradiction to the observed overprediction of the turbulence kinetic energy. The mechanism behind this observation is believed to be different for the downstream and the upstream movements of the pollutant.

When using the UDS model, the downstream movement of the pollutant is most likely insufficient because of an underestimate of the turbulent diffusion coefficient. Despite the overprediction of the turbulence kinetic energy, the turbulent diffusion coefficient $D_{t}$ as determined from Eq. 12 does not increase significantly compared to the simulations with the standard and the hybrid 1 settings, because the turbulence dissipation rate increases as well. The combination of this comparable diffusion coefficient and the decreased wake size results in an underprediction of the low concentration values further downstream for the hybrid 2 settings. For the DPM model, the downstream dispersion seems to be slightly better predicted, but the results in the low concentration region suffer from a large relative uncertainty because of the finite number of particles tracked. In addition, the particles seem to cluster in the low turbulence regions of the flow, which is a known non-physical effect of the discrete random walk model used for the calculation of the fluctuating velocity component (Fluent Inc. 2006).

The upstream movement of the pollutant is largely governed by the size of the lateral separation bubble. The hybrid 2 settings underpredict this separation bubble, and although this results in a better hit rate for the entire velocity field to the side of the building than the overprediction obtained with the standard and hybrid 1 settings, it strongly limits the upstream pollutant movement. As a result, the agreement in the concentration field is reduced. For the standard and the hybrid 1 settings the overprediction of the separation bubble size results in an overprediction of the upstream pollutant concentration when using the DPM model. When using the UDS model, an overprediction in the concentration values is most likely avoided because of an underestimation of the turbulent diffusion coefficient using Eq. 12.

\section{Conclusions and Further Work}

The results obtained for the flow field have shown that modifying the constants $C_{\mu}$ and $\sigma_{\varepsilon}$ to values that improve the streamwise homogeneity of the turbulence kinetic energy profile 
imposed at the inlet, affects the solution for the velocity and the turbulence kinetic energy field around the building. When using the modified constants in the entire computational domain, the overprediction of the wake length is reinforced as compared to simulations using the standard values. When restricting the modification to the region for which it was derived (the undisturbed ABL), this effect can be eliminated. Switching to the standard constants within the entire influence area of the building produces the best prediction for the wake length, but significantly overpredicts the turbulence kinetic energy. Switching to the standard constants only in the wake region results in a better prediction for the turbulence kinetic energy, but overpredicts the wake length. By varying the region in which the modified turbulence model constants are applied, it might be possible to obtain an improved solution in terms of both the velocity and the turbulence kinetic energy field.

When considering the concentration field, solving the transport equation for a scalar in combination with the hybrid 1 settings (modified turbulence model constants, but standard values in the building wake) and the Kato-Launder modification gives the best performance in terms of the hit rate. The hybrid 2 settings (modified turbulence model constants, but standard values within the building influence), which produce the best prediction of the velocity field, result in the lowest hit rate for the concentration field. For the present configuration, and considering particles that behave as a passive scalar, solving the transport equation for a scalar results in a better overall agreement with the test data (a higher hit rate) than applying a particle tracking model. When the implementation of a specific force is required, this conclusion might not hold, since it is not possible to include explicit formulations for additional forces in the scalar transport formulation.

It has been confirmed that satisfactory prediction of the velocity field is an important, but not sufficient, condition for obtaining a satisfactory prediction of the concentration field. The parameters that govern the turbulent dispersion need to be correctly predicted as well. For the DPM model, this requires an accurate prediction of the turbulence kinetic energy and a good estimate of the Lagrangian time scale. For the UDS model, a good estimate of the turbulent diffusion coefficient, which is usually defined as the ratio of the turbulent viscosity and the turbulent Schmidt number, is important. Ideally these parameters would be calculated based on satisfactory predictions for the turbulence field. The formulations for the Lagrangian time scale and the turbulent dispersion presented herein are a first step towards using flow field specific values instead of default values, or values obtained from optimising agreement with test data. The establishment of more accurate estimates, which could e.g. represent the anisotropic character of the turbulent dispersion, would most likely result in improved predictions of the concentration field.

In summary, further work should be dedicated towards obtaining a correct prediction of both the velocity and turbulence fields and towards obtaining accurate estimates of the Lagrangian time scale and turbulence diffusion coefficient based on this turbulence field. LES could provide useful information for both purposes. First, the settings of the turbulence model constants could be improved by extracting information on the required turbulent viscosity field that one should obtain from the RANS simulations. Secondly, LES would allow the calculation of the resolved turbulent diffusion coefficient and Lagrangian time scale and support the improvement of formulations for these quantities as a function of the turbulence field.

Acknowledgements This work was supported by IWT Vlaanderen, the Institute for the Promotion of Innovation by Science and Technology in Flanders, through the SBO project NanoSoc. We also thank the anonymous reviewers and the co-editor John Garratt for their valuable comments and suggestions. 


\section{Appendix}

\section{A.1 Uncertainty of the Non-Dimensional Velocity and Turbulence Kinetic Energy}

The uncertainty of a variable $X$, which is determined from a number of other variables $Y_{i}$, each having their own uncertainty, can be determined from:

$$
w_{X}=\sqrt{\sum_{i}\left(w_{Y_{i}} \frac{\partial X}{\partial Y_{i}}\right)^{2}} .
$$

For the non-dimensional streamwise velocity component $\langle U\rangle / U_{\text {ref }}$ the uncertainty can therefore be determined from the uncertainties of $\langle U\rangle, w_{\langle U\rangle}=0.07 \mathrm{~m} \mathrm{~s}^{-1}$, and $U_{\text {ref }}, w_{U_{\text {ref }}}=$ $0.1 \mathrm{~m} \mathrm{~s}^{-1}$ as given in the CEDVAL database. Within the lower velocity region, this uncertainty is larger than the relative allowed deviation of $25 \%$ used for the determination of the hit rate and can be approximated by a constant value of 0.012 .

For the non-dimensional turbulence kinetic energy $k / U_{\text {ref }}^{2}$, the uncertainty was determined by assuming $k=\frac{3}{2}\left\langle u^{\prime 2}\right\rangle$ with $\sqrt{\left\langle u^{\prime 2}\right\rangle}=T I \times\langle U\rangle$. As before, $w_{\langle U\rangle}=0.07 \mathrm{~m} \mathrm{~s}^{-1}$ and $T I$ is the turbulence intensity with $w_{T I}=7 \%$. When calculating the uncertainty of $\sqrt{\left\langle u^{\prime 2}\right\rangle}$ for the measurements, it was found that its value is close to $w_{\langle U\rangle}$ and the constant value of $0.07 \mathrm{~m} \mathrm{~s}^{-1}$ was therefore used in the calculation of the uncertainty of the non-dimensional turbulence kinetic energy. The resulting value for the uncertainty of $k / U_{\text {ref }}^{2}$ can be approximated by $0.0316\left(k / U_{\text {ref }}^{2}\right)^{0.518}$.

\section{A.2 Uncertainty of the Non-Dimensional Concentration}

The uncertainty of the non-dimensional concentration $K$ from the Lagrangian model is related to the tracking of a finite number of particles and was determined as a function of the average value of $K$ obtained from tracking five independent sets of 80,000 particle tracks. The uncertainty interval was calculated as 1.96 times the standard deviation, corresponding to a $95 \%$ confidence interval for a normal distribution. For values below $K=40$, this uncertainty was found to be higher than $25 \%$, which is the allowed relative discrepancy used for the determination of the hit rate (see Eq. 15). The uncertainty in this region is a function of the value of $K$ and can be approximated by $1.09 K^{0.63}$. This power law still implies zero uncertainty for $K=0$. Therefore, an additional criterion, allowing an absolute deviation for $K$ of $0.083\left(0.001 \%\right.$ of $\left.K_{S}\right)$, was adopted in the calculation of the hit rate.

\section{References}

Beranek W (1979) General rules for the determination of wind environment. In: Proceedings of the 5th international conference on wind engineering, pp 225-234

Blocken B, Carmeliet J, Stathopoulos T (2007a) CFD evaluation of wind speed conditions in passages between parallel buildings - effect of wall-function roughness modifications for the atmospheric boundary layer flow. J Wind Eng Ind Aerodyn 95:941-962

Blocken B, Stathopoulos T, Carmeliet J (2007b) CFD simulation of the atmospheric boundary layer: wall function problems. Atmos Environ 41:238-252

Blocken B, Stathopoulos T, Saathoff P, Wang X (2008) Numerical evaluation of pollutant dispersion in the built environment: comparisons between models and experiments. J Wind Eng Ind Aerodyn 96:18171831 
Castro I, Robins A (1977) The flow around a surface-mounted cube in uniform and turbulent streams. J Fluid Mech 79(2):307-335

Di Sabatino S, Buccolieri R, Pulvirenti B, Britter R (2007) Simulations of pollutant dispersion within idealised urban-type geometries with CFD and integral models. Atmos Environ 41:8316-8329

Donat J (1995) Windkanalexperimente zur ausbreitung von schwergasstrahlen. PhD thesis, University of Hamburg, Meteorological Institute, $179 \mathrm{pp}$

Fluent Inc. (2006) Fluent 6.3 Users's Guide, 2501 pp

Franke J, Hirsch C, Jensen A, Krus H, Schatzmann M, Westbury P, Miles S, Wisse J, Wright N (2004) Recommendations on the use of CFD in wind engineering. In: COST Action C14, Impact of wind and storm on city life built environment, von Karman Institute, pp C1.1-C1.11

Franke J, Hellsten A, Schlunzen H, Carissimo B (eds) (2007) Best practice guideline for the CFD simulation of flows in the urban environment-COST Action 732. COST office, $52 \mathrm{pp}$

Gao Y, Chow W (2005) Numerical studies on air flow around a cube. J Wind Eng Ind Aerodyn 93:115-135

Gorlé C, van Beeck J, Rambaud P, van Tendeloo G (2009) CFD modelling of small particle dispersion: the influence of the turbulence kinetic energy in the atmospheric boundary layer. Atmos Environ 43:673-681

Hargreaves D, Wright N (2007) On the use of the $k-\varepsilon$ model in commercial CFD software to model the neutral atmospheric boundary layer. J Wind Eng Ind Aerodyn 95:355-369

Kato M, Launder B (1993) The modeling of turbulent flow around stationary and vibrating square cylinders. In: Proceedings of 9th symposium on turbulence and shear flows, pp 1-6

Lakehal D, Rodi W (1997) Calculation of the flow past a surface-mounted cube with two-layer turbulence models. J Wind Eng Ind Aerodyn 67\&68:65-78

Leitl B (1998) Cedval at Hamburg University. http://www.mi.uni-hamburg.de/Introducti.433.0.html. Accessed 23 Jan 2010

Lim H, Thomas T, Castro I (2009) Flow around a cube in a turbulent boundary layer: LES and experiment. J Wind Eng Ind Aerodyn 97:96-109

Nakiboglu G, Gorlé C, Horváth I, van Beeck J, Blocken B (2009) Stack gas dispersion measurements with large scale-piv, aspiration probes and light scattering techniques and comparison with CFD. Atmos Environ 43:3396-3406

Oesterlé B (2006) Ecoulements multiphasiques—des fondements aux methodes d'ingénierie. Hermès, Lavoisier, $319 \mathrm{pp}$

Pope S (2008) Turbulent flows. Cambridge University Press, Cambridge, UK, 771 pp

Richards P, Hoxey R (1993) Appropriate boundary conditions for computational wind engineering models using the $k-\varepsilon$ turbulence model. J Wind Eng Ind Aerodyn 46(47):145-153

Riddle A, Carruthers D, Sharpe A, McHugh C, Stocker J (2004) Comparisons between FLUENT and ADMS for atmospheric dispersion modelling. Atmos Environ 38:1029-1038

Saathoff P, Stathopoulos T, Wu H (1998) The influence of freestream turbulence on nearfield dilution of exhaust from building vents. J Wind Eng Ind Aerodyn 77\&78:741-752

Schatzmann M, Leitl B (2002) Validation and application of obstacle resolving dispersion models. Atmos Environ 36:4811-4821

Shah K, Ferziger J (1997) A fluid mechanicians view of wind engineering: large eddy simulation of flow past a cubic obstacle. J Wind Eng Ind Aerodyn 67\&68:211-224

Tominaga Y, Stathopoulos T (2007) Turbulent Schmidt numbers for CFD analysis with various types of flowfield. Atmos Environ 41:8091-8099

Yang W, Jin X, Jin H, Gu M, Chen S (2007) Application of new inflow boundary conditions for modeling equilibrium atmosphere boundary layer in RANS-based turbulence models. In: Proceedings of international conference on wind engineering, pp 591-598

Yang W, Quan Y, Jin X, Tamura Y, Gu M, Gu M, Chen S (2008) Influences of equilibrium atmosphere boundary layer and turbulence model parameter on wind loads of low-rise buildings. J Wind Eng Ind Aerodyn 96:2080-2092

Yang Y, Gu M, Chen S, Jin X, Jin H, Gu M, Chen S (2009) New inflow boundary conditions for modelling the neutral equilibrium atmospheric boundary layer in computational wind engineering. J Wind Eng Ind Aerodyn 97:88-95 